\title{
Large-extended 2D supramolecular network of dipoles with parallel arrangement on a Si(111)-B surface
}

\author{
Irma Custovic, ${ }^{a}$ Damien Teyssieux, ${ }^{a}$ Judicaël Jeannoutot, ${ }^{a}$ Simon Lamare, ${ }^{\text {a }}$ Frank Palmino, ${ }^{\text {a }}$ Hamed Abbasian, ${ }^{\text {b }}$ Alain Rochefort \\ *b and Frédéric Chérioux*a
}

We have investigated the self-assembly of a strong dipolar molecule (LDipCC) on the semiconducting Si(111)-B surface with scanning tunneling microscopy (STM), density functional theory (DFT) calculations and STM simulations. Although the formation of an extended two-dimensional network was clearly revealed by STM under ultra-high vacuum, the assignment of a specific STM signature to the different terminal groups from the LDipCC molecular unit required a complete analysis by numerical simulations. The overall observed assembly is explained in terms of STM contrasts associated to the molecular structure of LDipCC and to the molecule-surface interactions. To distinguish the relative arrangement of the dipolar molecules within the assembly, a rational combination of experimental results and electronic structure calculations allow us to identify a single adsorbed LDipCC phase in which the molecular dipoles are homogenously arranged into a parallel fashion on the Si(111)-B surface.

\section{Introduction}

The interest for monolayered or ultra-thin ferroelectric materials containing highly dipolar molecules has significantly grown over the last two decades, this was highly motivated by the vigorous and recurrent needs for miniaturized devices. ${ }^{1,2,3,4}$ Such materials show very appealing properties that could be exploited in a broad range of applications such as random access memories, capacitors, sensors, and microactuators. $5,6,7,8$ In this context, monolayered supramolecular networks based on the adsorption of tailored dipolar molecules on surfaces are of increasing interests due to their abilities of being perfectly organized over a substantially large spatial domain.9,10,11,12,13,14 The successful development of extended supramolecular networks with dipolar molecules as building block on surfaces has been reported at very few occasions in the literature. Indeed, on metallic surfaces, the adsorption of dipolar molecules scarcely leads to the formation of large supramolecular networks but prevalently forms small molecular islands and multiple-domains where the orientation of the molecular units strongly depends on the magnitude and nature of the metal-molecule interactions. ${ }^{15,16,17,18,19}$ The self-assembly of dipolar molecules on metal surfaces is mostly dictated by the overall molecule-molecule interactions that limits the role of dipole-dipole interaction in the self-assembly process and seriously restrains the creation of a periodic network ${ }^{20}$. Nevertheless, few 2D extended networks have been successfully achieved on coinage metals by using a collaborative dipole-dipole interaction and intermolecular interaction. ${ }^{21,22}$ The role of dipolar interactions in a ferroelectric assembly has been elegantly demonstrated on Au(111) with the use of a heteroleptic double-decker molecule, in which one molecular moiety acts as the self-assembly agent on the surface while the upper moiety has a mobile dipole functionality that can easily rotate through a central metal-atom that unifies the molecule. ${ }^{23}$ In this case, the dipolar moieties are highly aligned into a ferroelectric fashion, and where the dipole orientation can be tuned under an electric field provided through a STM tip.

For semiconductor surfaces, role of the surface-molecule interaction is very important. For instance, in the case of the Si(001) surface, the deposition of dipolar molecules induces a reorganization of the surface charges in a fashion that promotes the formation of one-dimensional (1D) self-assemblies with periodic alignment of dipolar molecules over up to ten nanometers. ${ }^{24}$ This 1D arrangement is promoted by the template effect of the $\mathrm{Si}(001)$ due to its electronic structure. Therefore, the formation of extended two-dimensional (2D) ordered networks that exploit molecular dipole-like features requires the development of a more disruptive strategy where the magnitude of the dipole-dipole interactions should ideally become more significant than the molecule-surface contribution.

In this paper, we investigate the growth of 2D extended supramolecular network with periodic arrangement of a dipolar molecule on a $\mathrm{Si}(111)$-B surface. Our underlying objective is based on a tailored molecule that contains moieties leading to a self-assembly on the surface into a 2D network, and where the terminal groups that give a strong molecular dipole moment $\left(\mu_{D}=7.0 \mathrm{D}\right)$ moment can be aligned in one direction (parallel, PA) or in opposite direction (anti-parallel, AP). We report the formation of ordered and extended 2D supramolecular network of 1-(4"-cyanobiphenyl)-4-(4"-methoxybiphenyl)-2,5-bis(propyloxy)benzene (LDipCC) molecule on a Si(111)-B surface. Scanning Tunnelling Microscopy (STM), Density Functional Theory (DFT) calculations, which is a powerful tool to elucidate molecular adsorption on surfaces, ${ }^{25,26,27}$ and STM simulations were used to demonstrate that the LDipCC molecule is self-assembled into a highly homogeneous parallel arrangement on the Si(111)- $\mathrm{B}$ surface. In addition, Si(111)- $\mathrm{B}$ is a weakly reactive surface, anti-parallel arrangement has been mostly observed on $\mathrm{Cu}(111)$, but not on less reactive surfaces such as $\mathrm{Au}(111)$ and HOPG. ${ }^{15,16,17,18,19}$

\section{Results and discussion}




\section{A. Molecular engineering}

We have synthesized a 1-(4"-cyanobiphenyl)-4-(4"'-methoxy biphenyl)-2,5-bis(propyloxy)benzene (LDipCC) molecule as model of a strong dipolar molecule (See Figure 1a and ESI for synthetic procedure). This molecule contains a methoxy group (electron donor) and a cyano group (electron acceptor) distanced by $2.7 \mathrm{~nm}$ corresponding to the length of the five phenyl rings (see Figure 1a). In order to increase the solubility of LDipCC, we have added two short lateral propyloxy chains linked to the more central aromatic ring. These alkyl chains are long enough to improve the solubility of the molecule during the synthetic procedure. However, the alkyl chains are sufficiently short to limit the potential lateral van der Waals interactions between the molecular units among the assembly on the surface after deposition by sublimation.

\section{B. Deposition and self-assembly of LDipCC on a Si(111)-B surface}

The $\mathrm{Si}(111)$ - $\mathrm{B}$ has been deliberately chosen as a semiconducting surface archetype but where the saturation of the dangling bonds of silicon adatoms by underneath Boron (B) atoms is well-known to make them less reactive and offers a unique platform for the engineering of molecular patterns on this type of surfaces. ${ }^{6,28,29,30}$

Figure 1b shows an experimental STM image of a 0.9 monolayer coverage of LDipCC deposited onto the Si(111)-B surface at room temperature under UHV conditions and measured at $110 \mathrm{~K}$. As revealed in this image recorded at positive bias, no isolated molecule was observed but only large extended 2D islands of LDipCC (see ESI, Figure S5). The 2D networks are based on large domains, each one is constituted by the periodic arrangement of several bright protrusions. The basic unit cell is a parallelogram structure (vectors: $\overrightarrow{\boldsymbol{u}}$ and $\overrightarrow{\boldsymbol{v}}$ with $|\overrightarrow{\boldsymbol{u}}|=1.80 \mathrm{~nm}$ and $|\overrightarrow{\boldsymbol{v}}|=3.03 \mathrm{~nm}$ ) made from an eight bright spots pattern (see Figure $1 \mathrm{~b}$ ).

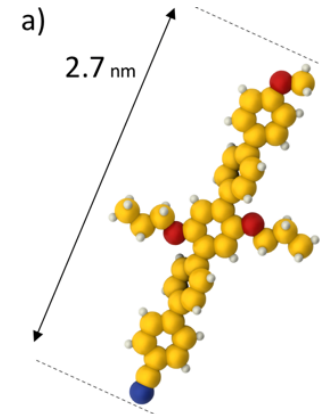

c)

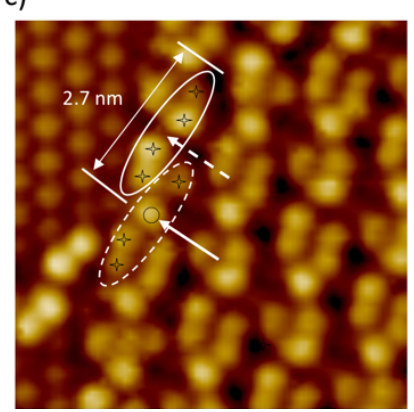

b)

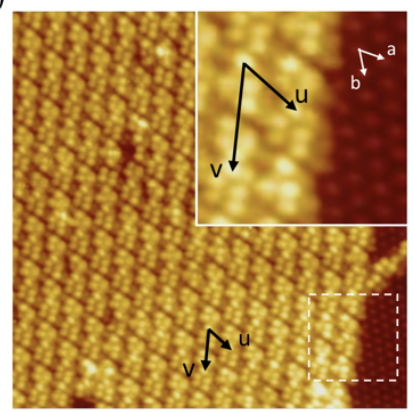

d)

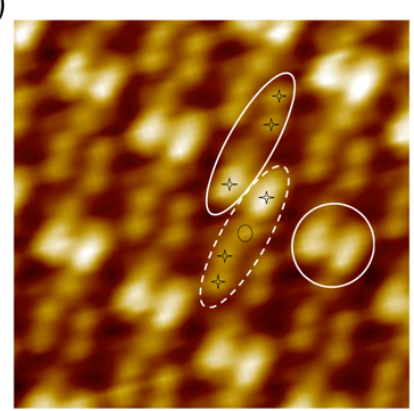

Figure 1. (a) Representation of the LDipCC molecule. Nitrogen atom: blue, Oxygen atoms: red, Carbon atoms: yellow and Hydrogen atoms: grey. (b) Large-scale STM image of a 0.9 monolayer of LDipCC on Si(111)-B surface with submolecular resolution on each molecule $\left(25 \times 25 \mathrm{~nm}{ }^{2}, V_{\mathrm{s}}=+2.3 \mathrm{~V}, l_{\mathrm{t}}=10 \mathrm{pA}, \mathrm{T}=110 \mathrm{~K}\right)$ and a zoom of step edge island as an inset. $U$ and $V$ are the vectors describing the unit cell of the supramolecular network, while a and $b$ are the lattice vectors of the $\mathrm{Si}(111)$ - $B$ surface. (c) High-resolution STM image of empty states $\left(7 \times 7 \mathrm{~nm}^{2}, V_{\mathrm{S}}=+2.3 \mathrm{~V}, I_{\mathrm{t}}=10 \mathrm{pA}, \mathrm{T}=110 \mathrm{~K}\right)$ of LDipCC/Si(111)-B near a step edge. Two different features are observed. The dashed-white ellipse surrounds a central bright protrusion (black circle) and three darker protrusions (black stars). The white ellipse surrounds four protrusions (black stars). (d) STM image of filled states $\left(7 \times 7 \mathrm{~nm}^{2}, V_{\mathrm{S}}=-1.4 \mathrm{~V}, I_{\mathrm{t}}=10 \mathrm{pA}, \mathrm{T}=110 \mathrm{~K}\right)$ of LDipCC/Si(111)-B. At this negative bias, each LDipCC molecule appears in a dissymmetric fashion: an elongated bright protrusion and three or four darker spots (black stars). The two bright elongated protrusions (large white circle) represent a side-by-side pair of LDipCC molecules.

High-resolution STM images in Figure 1c-d show regular domains of the LDipCC/Si(111)-B nearby a step edge of the molecular network. 
The repetitive unit pattern in STM images contains the two different features of similar length ( $2.7 \mathrm{~nm})$ compatible with the length of the molecule that are highlighted with ellipses in Figure 1c-d. Figure 1c shows the empty state. In this case, the full ellipse is made of four protrusions (identified by a star) separated by a more or less dark zone indicated by the dashed arrow. The neighbouring dashed ellipse also contains four separated protrusions but the brightest protrusion (black circle indicated by the white arrow) is at the center of the ellipse. For the two identified units, each protrusion is separated by a distance of $0.5 \mathrm{~nm}$. By probing the filled states through a negative bias, the relative brightness of the different protrusion significantly changes. The two different features described for Figure 1c appear now clearly dissymmetric along the molecule for negative bias (see Figure 1d) and the brightest protrusion of two adjacent features are now side-by-side (white circle in Fig 1d).

\section{Discussion}

A more detailed analysis of STM images at the submolecular level can now be performed and are detailed in Figure S6. Common features at positive and negative bias are directly related to the adsorption sites of the LDipCC molecule. LDipCC molecule appears mostly as four-protrusions in STM images at positive and negative bias voltage. For the molecules identified by a dashed-ellipse in Figure $1 \mathrm{c}$ and $1 \mathrm{~d}$, the more central aromatic ring (with two lateral propyloxy chains) sits over a silicon adatom (black circle) indicated by the white arrow in Figure 1c and Figure S6. For molecules within the lined-ellipses, the more central aromatic ring sits between silicon adatoms (dashed-arrow in Figure 1c). We have identified two LDipCC molecules per unit cell, that occupy an area of $4.9 \mathrm{~nm}^{2}$. Therefore, the molecular density is 1.2 molecules per $\mathrm{nm}^{2}$. In each domain, by using a matrix notation, this structure is described relatively to the lattice vectors $\overrightarrow{\boldsymbol{a}}$ and $\overrightarrow{\boldsymbol{b}}$ of the Si(111)-B surface as follows:

$$
\left(\begin{array}{cc}
2 & 1 \\
4 & -1
\end{array}\right)
$$

In each domain, the supramolecular network is commensurable with the $\mathrm{Si}(111)-\mathrm{B}$ surface and the epitaxy is of the type point-onpoint, supporting that molecule-surface interaction is stronger than molecule-molecule interaction. ${ }^{31}$

From the crystallographic analysis of the LDipCC arrangement on Si(111)-B based on STM images, we propose the two models of adsorbed LDipCC presented in Figure 2. The geometry of the individual LDipCC molecule corresponds to the optimized gas phase structure obtained by DFT (see ESI, Figure S7). We considered one alignment of the LDipCC molecular axis on the Si(111)-B surface where the terminal groups $\left(\mathrm{OCH}_{3}, \mathrm{CN}\right)$ are pointing toward similar (parallel or PA arrangement) or opposite direction (anti-parallel or AP arrangement).

From our STM observations and measurements, we can safely assign two types of features observed in Figure 1c-d to individual LDipCC molecule. Despite several attempts to identify an experimental STM signature for the terminal moities (by changing bias voltage, polarity, current, etc.), no specific signals allow us to clearly distinguish between the two molecular extremities (cyano versus methoxy groups). Nevertheless, the STM image of filled states (see Figure 1d) clearly shows that the STM contrasts localized on the terminal groups are reminiscent of a head-to-head configuration. No such clear feature was observed for empty states at positive bias beyond a well-organized distribution of STM contrasts among LDipCC units. Therefore, a more detailed study of the electronic structure of LDipCC was investigated with DFT and STM simulations in order to reveal the chemical nature and composition of frontier orbitals of adsorbed species.
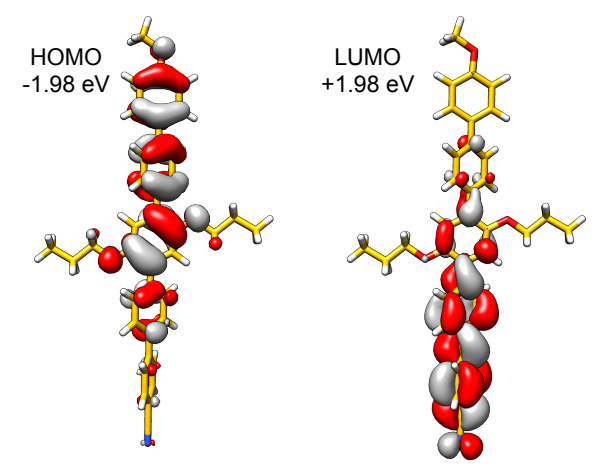

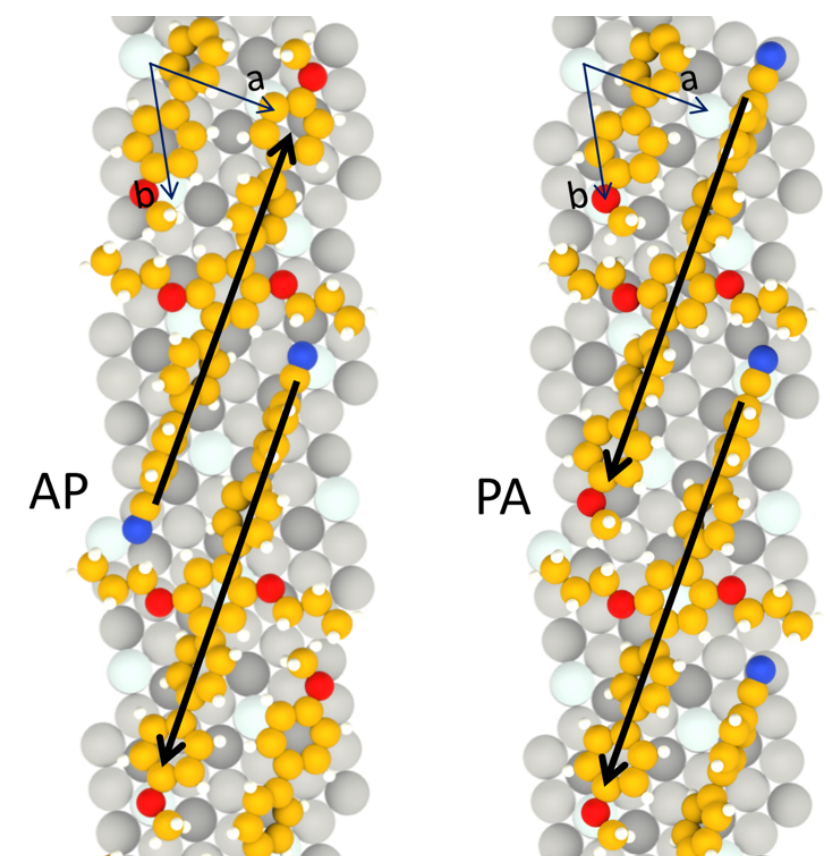

Figure 2. Proposed models for parallel PA (left panel) and anti-parallel AP (right panel) arrangement of $\mathrm{LDipCC} / \mathrm{Si}(111)-\mathrm{B}$. $\mathrm{a}$ and $\mathrm{b}$ are the vectors describing the surface lattice of $\mathrm{Si}(111)-\mathrm{B}$ surface.

A simple approach often used to better understand STM contrast is to perform DFT calculations on isolated molecular systems. Hence, we first determined the electronic structure of a single LDipCC molecule but also of a few small LDipCC islands assembled into PA and AP arrangements as in Figure 2. Figure 3 compares the nature of frontier orbitals (HOMO, LUMO) of a single gas phase LDipCC molecule. HOMO is mostly localized in the vicinity of the methoxy branch, whereas LUMO is centred among the cyano fragment. The small assembled PA and AP models, although not optimized, show highly discernible patterns similar to HOMO and LUMO (see ESI, Figure S8). HOMO/LUMO states are grouped in pairs in the AP conformation while they are well separated in space from a molecule to another in the PA arrangement. A priori, such wavefunction localization should constitute reliable signatures to discriminate both the molecular arrangement and the chemical nature of terminal groups. This result supports that the asymmetrical contrasts observed in Figure $1 \mathrm{~d}$ would be related to the AP model where the closest LDipCC pairs present similar head-to-head terminal groups.

Figure 3. Wavefunction contours of HOMO and LUMO for the LDipCC molecule. (isosurface $=0.02 \AA^{3}$ ). Energies are given with respect to mid-gap DFT energy defined to 0 eV. 
Those simple DFT calculations, in addition to the STM measurements, suggest that LDipCC molecules on the Si(111)-B surface could be well-organized into an anti-parallel fashion. While the paired contrasts observed for filled states could be associated to HOMO states in the AP phase, the STM contrasts observed experimentally for empty states do not show a distinct contrast inversion as calculated by DFT. Two factors could contribute to attenuate the anticipated STM contrasts. First, the presence of underneath silicon adatoms can significantly contribute to the STM contrasts, ${ }^{8}$ and more especially at positive bias. ${ }^{32}$ Second, since the energy barriers for the rotation of the different rings within a LDipCC molecule are only about a few $\mathrm{kcal} / \mathrm{mol}$, then the orientation of those rings with respect to the STM tip may have a significant influence on the resulting contrasts. ${ }^{33}$

(a)
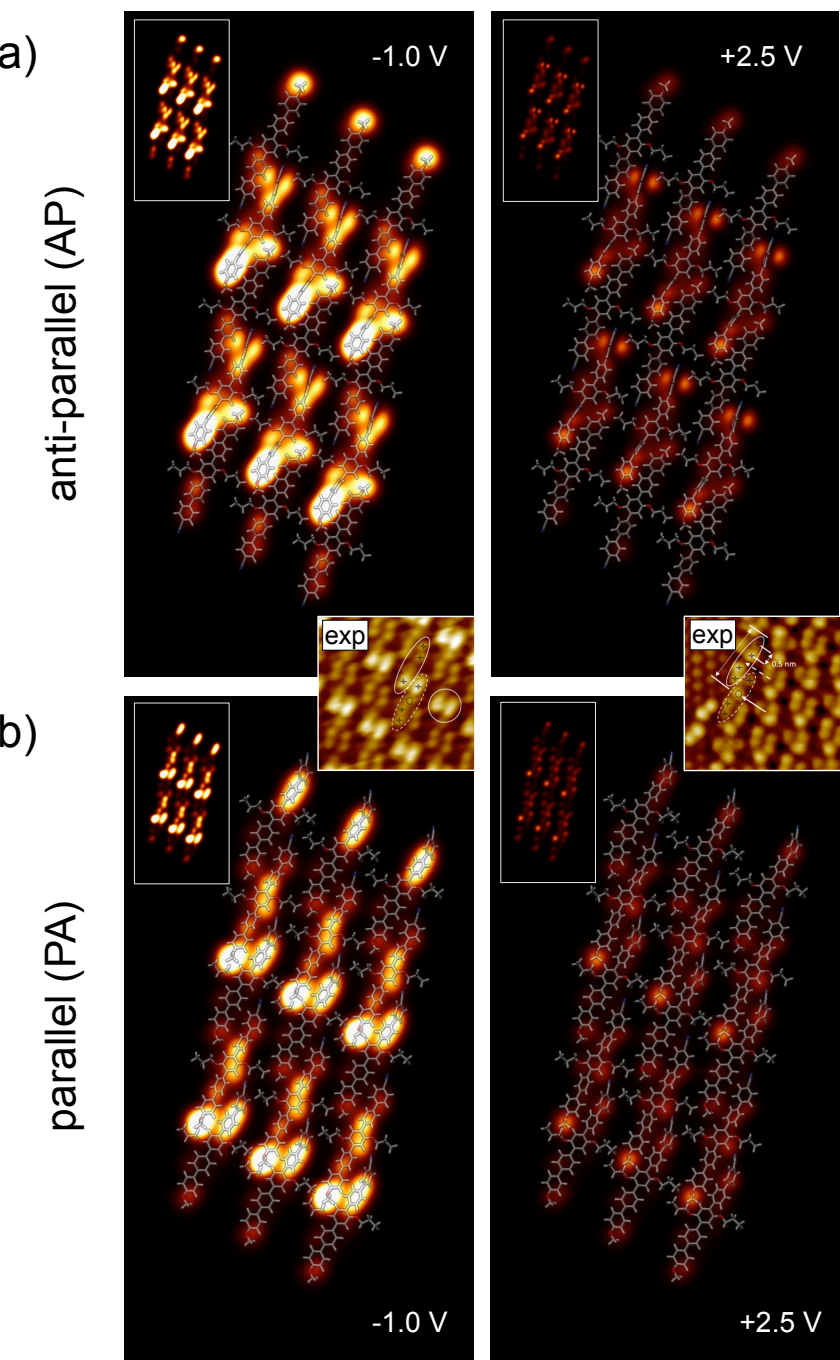

In order to overcome these two limiting factors, we perform additional DFT calculations on PA and AP arrangements by including the effect of the Si-B substrate. Models from the unit cells shown in Figure S9 were used to determine the optimized geometry of the PA and AP arrangements on the Si(111)-B surface (see ESI for more details and Figure S9).

As expected, the relaxation of LDipCC molecules in the PA and AP arrangement on $\mathrm{Si}(111)$-B mostly induced the rings rotation including a slight torsion of the most central ring to minimize the intermolecular interactions among the neighbouring rings and lateral chains. Despite we are fully aware that geometry optimization of such complex systems with high degree of freedom cannot systematically lead to a global energy minimum, our DFT results indicate that the PA arrangement is more stable than the AP by $0.15 \mathrm{eV} / \mathrm{unit}(-0.08 \mathrm{eV} /$ molecule). This value can be broken into $-0.85 \mathrm{eV} /$ unit for the molecule-surface interactions, and +0.70 $\mathrm{eV} /$ unit for the molecule-molecule interactions. Surprisingly, this last result predicts that free standing AP arrangement (but fixed at its optimized geometry on $\mathrm{Si}(111)-\mathrm{B})$ is more stable than PA. This energy difference reflects a complex balance of contribution from at least van der Waals, dipole-dipole and network energy that would necessitate a more extended study to identify the different contributions. Hence, our calculations clearly indicate that self-assembly of LDipCC on Si(111)-B is driven by moleculesurface interactions. Second, although we cannot accurately identify the magnitude of each intermolecular contributions at this stage, we note that the energy difference between the free-standing AP and PA phases agrees with a minimization of dipolar interactions in AP. We also understand that the energy balance between molecule-surface and molecule-molecule is relatively small, STM simulations on AP and PA arrangements should provide additional information. 
Finally, STM simulations on large-scale models of AP and PA arrangement containing 15 LDipCC molecules and the Si(111)-B substrate (see ESI for more details) are reported at Figure 4. For the sake of clarity, the optimized LDipCC assemblies without the $\mathrm{Si}-\mathrm{B}$ substrate are overlaid to the simulated images in the main panels, and the different insets give the calculated and experimental STM images. The STM images of occupied states (left panels) were calculated for a bias of -1.0 V, while the STM image of empty states (right panels) were evaluated at a bias of $+2.5 \mathrm{~V}$. The bias used in the STM simulations are defined with respect to Fermi level of the LDipCC/Si(111)-B system that is nearby the HOMO shown at Figure 3 and located among the calculated LDipCC band gap. The difference in bias voltage used in the STM simulations ( $3.5 \mathrm{~V})$ is in the same range than the experimental conditions used (3.7 V).

Figure 4. STM simulations on large-scale models of LDipCC assembled into (a) anti-parallel and (b) parallel arrangements. The STM models were built from the optimized unit cell obtained from DFT. The models consist of a substrate made of a $3 \times 3 \mathrm{Si}(111)-\mathrm{B}$ unit cell as in Figure 2, and an adsorbed assembly containing 15 LDipCC molecules. Simulated STM images where calculated for a constant height, and include the states contained between the Fermi level $\left(\mathrm{E}_{\mathrm{F}}=0 \mathrm{~V}\right)$ and $+2.5 \mathrm{~V}$ for empty states (right panels) and $-1.0 \mathrm{~V}$ for filled states (left panels). The same current scale was used for positive and negative bias. The corresponding experimental images (exp) are shown for comparison.

A rapid comparison between calculated STM images at negative and positive bias for both AP an PA does not clearly reveal the contrast inversion we have previously identified from the nature of HOMO and LUMO of isolated LDipCC. The most striking feature of experimental STM images for filled states $(\mathrm{V}<0)$ (Figure $1 \mathrm{~d}$ ) is the head-to-head pairs of contrasts that is somehow reproduced in the simulated image of the PA model (Figure 4(b), left panel). From a general perspective, the STM contrasts appear welldistributed along a single molecular unit, and the resulting STM images look more continuous for the PA arrangement than for AP. Otherwise, a comparison of experimental and simulated STM images based on the relative spot positions gives a better agreement with the PA than the AP arrangement. As mentioned above, the relative rotation of rings within a single LDipCC unit may have a significant influence on the resulting STM intensities.

Hence, experimental and theoretical results agree to confirm the formation of an extended network based on a highly dipolar LDipCC molecule where the units are aligned in parallel fashion. In principle, such parallel arrangement should not minimize the lateral dipole-dipole interactions, our DFT calculations in fact predict that the free-standing AP is more stable than the PA phase. In fact, both $-\mathrm{CN}$ and $-\mathrm{OCH}_{3}$ groups are electron rich groups (see Figure $\mathrm{S7}, \mathrm{ESI}$ ) that provide local dipole pointing in opposed direction, but where the net dipole moment of the entire molecule is directed toward the $-\mathrm{OCH}_{3}$ group. More importantly, the molecule-surface interactions in the PA arrangement are stronger than the molecule-molecule interactions in the AP or PA phase. Eventually, LDipCC molecules are self-assembled over significantly large 2D-domains, which are commensurable with the Si(111)B surface thanks to the strong molecule-surface interactions and that essentially contain highly homogeneous parallel molecular arrangement, due to contribution of weaker molecule-molecule interaction.

\section{Conclusions}

In this article, we have demonstrated the formation of a 2D network based on dipolar molecules into a parallel arrangement on the Si(111)-B surface. In contrast to previous works, we observed significantly large arrays of LDipCC molecules where the individual dipoles form a homogenous parallel phase. The lateral dipole-dipole interactions could be reduced through the internal LDipCC charge distribution, even if the molecule-surface is the stronger than molecule-molecule interactions, as emphasized by the commensurability of the 2D supramolecular network with the Si(111)-B surface. Such results open a promising pathway to the development of nanodevices based on the self-assembly of dipolar molecules with atomically-precise organization for the development of devices based on future monolayered ferroelectric material.

\section{Conflicts of interest}

There are no conflicts to declare.

\section{Acknowledgements}

This work was supported by a grant from the Agence National de la Recherche (ACTION, ANR-15-CE29-0005) and a grant from the Pays de Montbéliard Agglomération. A.R. acknowledges the support from the Natural Sciences and Engineering Research Council of Canada (NSERC), and he is grateful to Calcul Québec and Compute Canada for providing computational resources. The authors acknowledge C. Krzeminski and G. Copie (IEMN-France) for fruitful discussions.

\section{Notes and references}


1 N. Nuraje and K. Su, Nanoscale, 2013, 5, 8752.

2 S. Yuan, X. Luo, H. L. Chan, C. Xiao, Y. Dai, M. Xie, and J. Hao, Nat. Commun., 2019, 10, 1775.

3 M. B. Avinash and T. Govindaraju, Acc. Chem. Res., 2018, 51, 414.

4 A. V. Gorbunov, M. G. Iglesias, J. Guilleme, T. D. Cornelissen, W. S. C. Roelofs, T. Torres, D. Gonzalez-Rodriguez, E. W. Meijer and M. Kemerink, Sci. Adv., 2017, 3, e1701017.

5 A. S. Tayi, A. Kaeser, M. Matsumoto, T. Aida and S. I. Stupp, Nat. Chem., 2015, 7, 281.

6 N. Setter, D. Damjanovic, L. Eng, G. Fox, S. Gevorgian, S. Hong, A. Kingon, H. Kohlstedt, N. Y. Park, G. B. Stephenson, I. Stolitchnov, A. K. Taganstev, D. V. Taylor, T. Yamada and S. Streiffer, J. Appl. Phys., 2006, 100, 051606.

7 L. W. Martin and A. M. Rappe, Nat. Rev. Mater., 2016, 2, 16087.

8 Y.-Y. Tang, P.-F. Li, W.-Q. Liao, P.-P. Shi, Y.-M. You and R.-G. Xiong, J. Am. Chem. Soc., 2018, 140, 8051.

9 S. Clair and D. G. De Oteyza, Chem. Rev., 2019, 119, 4717.

10 Y. Makoudi, J. Jeannoutot, F. Palmino, F. Chérioux, G. Copie, C. Krzeminski, F. Cléri and B. Grandidier, Sur. Sci. Rep., $2017,72,316$.

11 L. Bartels, Nat. Chem. 2010, $2,87$.

12 J. A. W. W. Elemans, S. Lei and S. De Feyter, Angew. Chem. Int. Ed., 2009, 48, 7298.

13 A. G. Slater, L. M. A. Perdigao, P. H. Beton and N. R. Champness, Acc. Chem. Res., 2014, 47, 3417.

14 L. Sosas-Vargas, E. Kim and A.-J. Attias, Mater. Horiz., 2017, 4, 570.

15 T. Yokayama, T. Takahashi, K. Shinozaki and M. Okamoto, Phys. Rev. Lett., 2007, 98, 206102.

16 Y. L. Huang, R. Wang, T. C. Niu, S. Kera, N. Ueno, J. Pflaum, A. T. S. Wee and W. Chen, Chem. Commun., 2010, 46, 9040.

17 L. Xu, B. Zha, X. Miao and W. Deng, J. Phys. Chem. C, 2012, 116, 1061.

18 A. E. Baber, S. C. Jensen and E. C. H. Sykes, J. Am. Chem. Soc., 2007, 129, 6368.

19 T. K. Shimizu, J. Jung, H. Imada and Y. Kim, Angew. Chem. Int. Ed., 2014, 53, 13729.

20 D. A. Kunkel, J. Hooper, S. Simpson, D. P. Miller, L. Routaboul, P. Braunstein, B. Doudin, S. Beniwal, P. Dowben, R. Skomski, E. Zurek and A. Enders, J. Chem. Phys., 2015, 142, 101921.

21 J. V. Barth, J. Weckesser, G. Trimarchi, M. Vladimirova, A. De Vita, C. Cai, H. Brune, P. Günter, K. Kern, J. Am. Chem. Soc., 2002, 124, 7991.

22 A. Schriffin, A. Riemann, W. Auwärter, Y. Pennec, A. Weber-Bargioni, D. Cvetko, A. Cossaro, A. Morgante, J. V. Barth, PNAS, 2007, 104, 5279.

23 Y. Zhang, H. Kersell, R. Stefak, J. Echevarria, V. Lancu, U. G. E. Perera, Y. Li, A. Deshpande, K.-F. Braun, C. Joachim, G. Rapenne and S.-W. Hla, Nat. Nanotechnol., 2016, 11, 706.

24 K. R. Harikumar, T. Lim, I. McNab, J. C. Polanyi, L. Zotti, S. Ayissi and W. A. Hofer, Nat. Nanotechnol., $2008,3,222$.

25 C. Jing, B. Zhang, S. Synkule, M. Ebrahimi, A. Riss, W. Auwärter, L. Jiang, G. Médard, J. Reichert, J. V. Barth, A. C. Papageorgiou, Angew. Chem. Int. Ed., 2019, 58, 18948.

26 M. Fritton, D. A. Duncan, P. S. Deimel, A. Rastgoo-Lahrood, F. Allegretti, J. V. Barth, W. M. Heckl, J. Björk, M. Lackinger, J. Am. Chem. Soc., 2019, 141, 4824.

27 B. Verhlac, N. Bachellier, L. Garnier, M. Ormaza, P. Abbufager, R. Robles, M.-L. Bocquet, M. Ternes, N. Lorente, L. Limot, Science, $2019,366,623$.

28 S. R. Wagner, B. Huang, C. Park, J. Feng, M. Yoon and P. Zhang, Phys. Rev. Lett., 2015, 115, 096101.

29 B. Baris, J. Jeannoutot, V. Luzet, F. Palmino, A. Rochefort and F. Cherioux, ACS Nano, 2012, 8, 6905.

30 B. Baris, V. Luzet, E. Duverger, P. Sonnet, F. Palmino and F. Cherioux, Angew. Chem. Int. Ed., 2011, 50, 4094.

31 D. E. Hooks, T. Fritz and M. D. Ward, Adv. Mater., 2001, 13, 227.

32 I. W. Lyo, E. Kaxiras and Ph. Avouris, Phys. Rev. B, 1989, 63, 1261.

33 C. J. Chen, Introduction to Scanning Tunneling Microscopy, $2^{\text {nd }}$ edition, Oxford University Press, 2008. 